ON THE RECORD

CIsolating stem cells

from the placenta is not more difficult than making a steak."

Theeditors of online 'how-to' guide makezine.com, in a blog entry on the joys of isolating your own amniotic stem cells at home.

\section{SCORECARD}

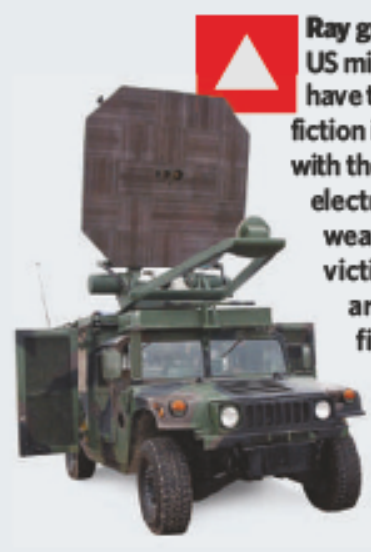

\section{Ray guns}

US militar y researchers have turned science fiction into reality with their 'harmless' electromagnetic weapon that makes victims feel as if they are about to catch fire. This non-lethal way to disarm people could help save the lives of civilians and army personnel

in battle zones such as Iraq, Pentagon officials claim.

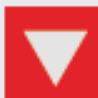

Space tourism 'Lucky' competition winner Brian Emmett has reluctantly givenup his seat on a flightinto space with cosmic tour operator Space Adventures after realizing that the prize, offered by software company Oracle, would come with a $\$ 25,000$ tax bill.

\section{NUMBER CRUNCH}

$110 \%$ is how much the price of US home heating oil rose between 2000 and 2007.

$\mathbf{8 2} \%$ is how much petrol prices rose during the same period.

$357 \%$ is how much ExxonMobil's profits grew between 2000 and 2006.

\section{ZOO NEWS}

The owners of a Malaysian fruit orchard have discovered why their guard dogs kept disappearing - a 7.1-metre python had eaten 11 of them before being captured by villagers.

Sources: Makezine.com CNN, Los Angeles Times, Campaign for America's Future, Reuters

\title{
Physicists plan search for the known unknowns
}

Gravity feels like a force you can trust. Every day, unwavering, it keeps your feet planted firmly on the ground.

But for many physicists, gravity is unsettling. It's the one force that doesn't fit into their quantum picture of the world. The best theory to describe it - Einstein's general theory of relativity - doesn't mesh with quantum mechanics.

What's more, general relativity breaks down in the singularities thought to lurk at the centre of black holes, suggesting to physicists that the theory is incomplete. So last week at the University of Arizona in Tucson, around 50 physicists met at a workshop called 'rethinking gravity', to confront these problems.

Over three days, participants debated what experiments they could use to hunt for flaws in the general theory of relativity, and asked whether dark matter or dark energy - the unknown stuff that cosmologists now reckon makes up $96 \%$ of the Universe - are clues to whatever deeper theory lies beneath.

Their proposals stretch from a table-top experiment so delicate that it can be sent offkilter by a single piece of dust, to ambitious space missions to measure the gravitational ripples created when two black holes coalesce into one.
Theoretical progress in combining gravity and quantum mechanics has produced ideas such as string theory and loop quantum gravity. And although no theory has yet been proposed that physicists accept as compelling, efforts to develop alternatives have been bolstered by recent discoveries in cosmology.

Maps of the temperature of the cosmic microwave background, for example - the radiation left over from the birth of the Universe - have revealed two opposing influences on the Universe's structure. One helps matter to clump together, the other accelerates the expansion of the Universe. Taken with other evidence, these observations suggest the existence of dark matter and dark energy, respectively.

A modified theory of gravity might account for the effects - although not everyone is convinced that it will do so. "It's very easy to sit around in the coffee breaks and say, maybe there is no dark stuff and gravity is different," warns Sean Carroll of the California Institute of Technology in Pasadena, who works on alternative theories to produce expansion like that seen with dark energy. "But if you sit down to actually do something, there are rules you have to follow. It's harder than you might think"

\section{On the launch pad}

Five astrophysics projects are competing to be the first mission from NASA's 'Beyond Einstein' programmeto fly. Conceived in 2002, the programme aims to address fundamental questions in cosmology (see Nature 420,593-594; 2002), and could help physicists in their understanding of gravity.

The five ideas are being reviewed by a panel convened by the US National Academies at the request of NASA and the Department of Energy. Mission teams presented their cases at a meeting in Newport Beach, California, this week, in their second meeting with the panel.
Of the two major missions under consideration, one will be built and operated jointly with the European Space Agency. Called the Laser Interferometer Space Antenna, or LISA, the project willsearch for gravitational waves.

The other large-scale proposal is Constellation- $X$, a powerful $X$-ray telescopeto study the processes around blackholes.

The three smaller proposals are the Joint Dark Energy Mission, being developed jointly by NASA and the Department of Energy; an inflation probe that will try to determine how the Universe expanded after its birth; and a probe to detect black holes.

The panel will consider the technical and management challenges as well as the missions' scientific merit. It is expected toreport in September, to assist with NASA's funding plans for 2009.

Someworry that the missions face an uncertain future if they miss the top spot. But Michael Salamon, programme manager for Beyond Einstein, says that all of them will eventuallygo ahead. "NASA is committed to the entire Beyond Einstein programme", he says.

"There is no 'winner takes all" scenario here." 